\title{
Management of Electronic and Electrical Quality Inspection at the Angle of Just and Public Trust
}

\section{Zhou Jiefei}

\section{Science and Technology Supervision Bureau, Wen'an County, Hebei Province, Langfang 065800}

Abstract: With the rapid development of society, people pay more attention to the problem of energy conservation, environmental problems are also the world to solve the problem. Today, in the perspective of impartiality to strengthen the quality of electrical and electronic testing and management can not only better guarantee the quality of electrical and electronic, but also can reduce energy consumption and improve energy efficiency. In order to strengthen the electrical and electronic quality inspection work management, energy conservation, the state issued the following laws and regulations: First, "Energy Conservation Law", the second is "Electronic and Electrical Products Quality Law", the third is "product quality certification and accreditation regulations." However, the quality of electrical and electronic testing must adhere to the "fair" "public letter" principle, in order to play the role of electronic and electrical quality testing.

Key words: impartiality; electronic appliances; quality inspection; management

\section{Introduction}

Although China is a large energy country, but the energy per capita share is very low ${ }^{1}$. In the perspective of impartiality to strengthen the electrical and electronic quality inspection work management, can reduce energy consumption and promote national economic development. To achieve the electrical and electronic quality inspection work management objectives, we must combine the actual situation to develop electronic and electrical quality inspection work management program, to achieve effective management.

1. The content of electronic and electrical quality inspection from the perspective of impartiality
Energy efficiency testing is the key link of electrical and electronic quality inspection. The so-called energy efficiency test is actually the ratio between the energy and the energy used in the electronic appliances $^{2}$. For example, the energy efficiency of the induction cooker and the induction cooker which people often use at home is actually the induction cooker the energy produced after the energy and the energy consumed in the operation. For example, in the detection of people commonly used induction cooker energy efficiency, the inspector first need to detect the voltage of the induction cooker, and then detect the specific heat capacity of the induction cooker. For the rice cooker energy efficiency testing, including the following: First, the quality of empty rice cooker, the second is the quality of water, the third is the initial temperature of water, the fourth is the energy consumption of rice cooker boot, the fifth is the rice cooker signal processing. For the washing machine energy efficiency testing, including: First, the washing machine water consumption, the second is the washing machine power consumption, the third is the washing machine washing performance, four washing machine dehydration performance. For air-conditioning energy efficiency testing, including: First, the temperature of air conditioning and refrigeration, the second is the air conditioning cooling flow, the third is the pressure when the air conditioning wet. In the perspective of impartiality, electronic and electrical quality testing has the following characteristics: First, comprehensive, the second is the complexity. In fact, to carry out the work of electrical and electronic quality testing process, a variety of factors will affect the authenticity and accuracy of test data, which are for the quality of electrical and electronic testing work management put forward higher requirements. 
2. The implementation of electrical and electronic quality inspection work management at the angle of fair and public trust

The state in order to improve the utilization of energy, combined with the actual situation of the domestic development of the energy labeling system. "Electronic and electrical products directory" contains the following: First, the electrical and electronic energy efficiency labeling, the second is the implementation of the rules, the third is the energy efficiency labeling style, four electronic and electrical specifications. "Electronic and electrical products catalog" contains the contents are described in detail, and must be authorized by the relevant state departments, do the record. Imported electrical and electronic products energy efficiency can be detected by a professional testing organization, can also be detected by the relevant state departments, combined with domestic energy efficiency standards to determine the energy efficiency of electronic products. In this regard, China's electrical and electronic products, energy efficiency testing agencies must continue to improve the detection environment, improve the professionalism and comprehensive quality of testing personnel, and better ensure that the electrical and electronic products, energy efficiency test data authenticity and accuracy to meet the electrical and electronic energy efficiency testing needs, improve the level of product energy efficiency testing ${ }^{3}$. National regulatory authorities must also play a role, play the role of supervision and management, and regulate the electrical and electronic energy efficiency testing agency behavior.

\section{Analysis of Quality Inspection Technology of Electronic and Electrical Apparatus from the Perspective of Fair and Public Trust}

\subsection{Analysis of internal quality inspection technology of electronic and electrical appliances}

In the perspective of fairness and credibility, electronic and electrical quality testing work cannot be carried out blindly, must be combined with the actual situation to develop electronic and electrical quality testing program, a clear electrical and electronic quality testing objectives. Electronic and electrical quality inspection must be carried out by the professional testing department. Electronic and electrical quality testing include: First, internal quality testing, and second, external quality testing. Before carrying out the internal quality inspection of electrical and electronic equipment, the testing organization needs to do the following preparatory work: First, improve the detection mechanism of the detection configuration. Electronic and electrical products quality inspection agencies must have the test qualification, but also to have a detection mark, the detection mechanism of the environment and capacity must also meet the relevant state regulations. Electronic and electrical products quality inspection agencies must have: First, a professional testing equipment, the second is a professional testing staff, the third is a reasonable test specifications. Only with the detection capability of the testing institutions can carry out electrical and electronic product quality testing work.

In fact, the authenticity and accuracy of electronic product quality testing data by many factors, mainly including the following factors ${ }^{4}$ : First, detection technology, the second is the detection staff, the third is the detection equipment. In this regard, the electrical and electronic quality inspection agencies must introduce advanced testing equipment, and focus on testing equipment maintenance and maintenance, to extend the service life of testing equipment. Testing agencies also need to focus on testing personnel training, and constantly improve the professionalism of the testing staff and comprehensive quality, and thus improve the detection level.

\subsection{Electronic and electrical external quality testing technology analysis}

To improve the quality of China's electrical and electronic products, the level of external quality testing, China needs a reasonable reference to the Western testing organizations testing experience, shorten the gap between the Western countries, keeping up with the pace of the times. China's testing organizations also need to sum up the problems in the detection of various aspects, and a detailed analysis of the reasons for the existence of the problem in order to timely detection of the problems taken to adjust the countermeasures. Electronic and electrical product quality testing has become the most important task of testing, the country must focus on the issue.

\section{Conclusion}

Today, people on the electrical and electronic quality testing work continues to improve the degree of attention, electronic and electrical quality testing is not only related to the quality of electrical and electronic, but also related to people's quality of life. In this regard, the relevant state departments must recognize the importance of electronic and electrical 
quality testing, combined with China's electronic and electrical market characteristics and the actual situation to develop testing programs, planned, targeted to carry out electrical and electronic quality testing, not blind. In the process of carrying out electrical and electronic quality testing, many factors will affect the accuracy of electronic and electrical quality inspection data. In this regard, the quality of electrical and electronic testing agencies must start to ensure that the quality of electrical and electronic testing data authenticity and accuracy, improve the detection level, play the role of testing agencies. In the process of conducting electrical and electronic quality testing, the professionalism and overall quality of the testing personnel have a great influence on the accuracy of the test data. In this regard, the testing agencies must recognize the importance of strengthening the training of personnel testing, training personnel to increase the training of personnel investment, improve the professional level

of testing and comprehensive quality, and thus improve the level of electronic and electrical product quality testing. Testing agencies must also continue to optimize the electrical and electronic product quality testing environment, better meet the electrical and electronic product quality testing needs.

\section{References}

[1] Li Ling. Justice and Credibility of Electronic and Electrical Quality Inspection Work Management [J]. Electronic production, 2017, (06): 96-97.

[2] Pan Tie. Electronic Commerce Product Information Standard Conformance Test Platform Design and Implementation [D]. Beijing University of Technology, 2016.

[3] Wang Junping. Perishable Goods Supply Chain Optimization and Coordination Issues [D]. Hefei University of Technology, 2014.

[4]Qing Yuan, Wu Yuzhe. Quality control technology for energy efficiency inspection of electronic and electrical products $[\mathrm{J}]$. Technology Innovation and Application, 2017,(24):43+45. [2017-09-22]. 